read at the symposium will be published in full in a separate volume by the Kazak Academy of Sciences during 1968. Another symposium, with a similar purpose, will be held in two years' time.

\section{Nutrition for the Old}

"Nutritional Problems of the Elderly" was the subject of a symposium held by the Scottish Group of the Nutrition Society in Edinburgh on April 20. Professor W. F. Anderson, the chairman, referred to surveys now being made in the hitherto neglected field of the nutrition of elderly people. Recent studies have indicated that food intake, noticeably of fat, diminishes in the seventh and eighth decades. The intakes of essential food substances which are often low in the elderly are those of vitamin $\mathrm{C}$, thiamine, folic acid, vitamin $B_{12}$ and iron, but definite nutritional deficiency diseases are rare. Minor deficiency conditions probably exist but are not recognized. Elderly persons living at home tend to have superior levels of these nutrients compared with those living in hospitals. In the latter group, vitamin $\mathrm{C}$ was particularly low and normal hospital diets appear to need close supervision in this respect.

Dr J. V. G. A. Durnin of the Institute of Physiology, University of Glasgow, speaking on "Energy Requirements, Intakes and Balances", pointed out that for most adults, occupational activity accounts for the greater part of their physical energy expenditure and leisure activities, often sedentary, for the remainder. The elderly are generally at leisure and few are really fit and active. These factors mean that most of this group will expend physically only small quantities of energy. Changes in body composition have been noted with lean people over 65 years old. This may be caused by reduced calorie intakes observed in the elderly or to shorter life expectation in the obese. In studies on absorption, significant deterioration for ages up to 75 years has not been found.

"The Protein Status of the Elderly" was discussed by Dr W. T. C. Berry of the Ministry of Health, London. At present, methods of detecting the mild effects of protein deficiency in the individual are not very sensitive and knowledge is insufficient to enable a borderline between the adequate and the inadequate state to be well defined. To distinguish between normal signs of ageing and the milder signs of malnutrition there is the problem as to what evidence should be sought. Reduced serum albumin levels, which may accompany shrinkage of muscle, may precede obvious signs of protein deficiency. The relationship between dietary protein intake and these changes is being investigated.

Speaking on "Dietary Hazards of the Elderly", Dr J. Andrews of the West Middlesex Hospital, Isleworth, said that in his experience the three groups of patients most vulnerable to malnutrition are those with gross locomotor disease which may serve to isolate them, those who are mentally disturbed and those in institutions. Protein and vitamin D were low in these out-patients. Many cases of iron deficiency anaemia were due to causes such as previous gastro-intestinal operations, and cases due solely to malnutrition were not found.

Dr D. A. Smith of the Gardiner Institute, Western Infirmary, Glasgow, spoke on the falling mineral content of bone with age and the high incidence of osteoporosis as a positive major threat, particularly to the elderly woman. As many as one in five old women may become osteoporotic. For men the risk is less.

\section{Chain Termination}

\section{from our Cell Biology Correspondent}

CAPECCHI at Harvard is using the RNA from an amber mutant of the bacteriophage $R 17$, which is at the position of the sixth amino-acid in the phage coat protein and directs the synthesis of a hexapeptide in vitro, to study chain termination (see Nature, 216, 226; 1967). At Cambridge, Bretscher is using an identical mutant of the $f 2$, originally isolated by Zinder's group, for the same purpose. Neither of them has yet found how chain termination is achieved, but Capecchi seems to have isolated an enzyme involved in termination, and now Bretscher has proved that the termination codon is read. Termination is therefore an active process, not simply the result of inability to read a codon. Furthermore, both workers have established that the terminating codon is not read by a $t$ RNA similar to the $t$ RNA molecules that read all the other codons.

Bretscher's experiment is admirably direct. He has taken the RNA from wild type $f 2$ and from the position six amber mutant and used it as messenger in a cellfree system. The cell-free system contains ribosomes and supernatant fraction but only six highly purified species of $t \mathrm{RNA}$ - those specific for the six amino-acids in the hexapeptide-in other words, the first six aminoacids in the phage coat protein, and therefore the system is able to translate up to, but not beyond, the position of the amber mutant.

$\mathrm{He}$ asks whether in these conditions a terminated hexapeptide free of $t$ RNA is produced by both messenger RNAs or only by the mutant RNA containing the amber chain terminating codon. The wild type RNA in fact programmes the synthesis of a hexapeptidyl$t$ RNA. The mutant RNA programmes the synthesis of a free hexapeptide detached from $t$ RNA. Thus when translation is brought to a halt by the absence of a $t$ RNA molecule, termination-the release of the peptide free from all translating machinery-does not occur unless there is a chain terminating codon. What is more, because only six specific $t$ RNAs were used and, like Capecchi, Bretscher finds that termination cannot be prevented by destroying all the other $t$ RNAs not involved in the synthesis of the hexapeptide, it appears that there is no $t \mathrm{RNA}$ for reading chain terminating codons. What then reads UAG, UAA and UGA with sufficient accuracy not to confuse them with other codons? Is it the protein Capecchi has isolated ?

\section{Sex Hormones and Cancer}

\section{from our Medical Biochemistry Correspondent}

IT is now well known that cancers of the breast and prostate are often influenced in their growth by variations in the concentrations of sex hormones in the blood, and that excretion of sex hormones is significantly increased in patients with cancers of the breast and prostate (Science, 145, 716; 1964). It is rather more surprising to find that the plasma concentrations 